\title{
ROTOR FLUX OPTIMAL ESTIMATION FOR INDUCTION MOTOR CONTROL
}

\author{
F. Alonge, F. D'Ippolito, G. Giardina, F. M. Raimondi, T. Scaffidi
}

Dipartimento di Ingegneria dell' Automazione e dei Sistemi

University of Palermo, Palermo, Italy

\begin{abstract}
The aim of this paper is to analyze and design reduced order observers of the rotor flux of induction motors. The design requirements are: a) the convergence rate of the rotor flux estimation error; b) a low sensitivity to stator and rotor resistance variations; c) a low sensitivity to errors due to the implementation of the observers on microprocessor-based systems. It is shown that, in order to satisfy the requirements a)-c), it is sufficient to solve a constrained optimization problem according to a criterion in which these requirements appear explicitly. The implementation of the observer is discussed. The observer is tested by simulation and experiments. Copyright (C) 2005 IFAC
\end{abstract}

Keywords: Induction Motors, Reduced Order Observers.

\section{INTRODUCTION}

As is well known, the implementation of a control law based on rotor flux control for asynchronous motors implies the estimate of modulus and phase of the rotor flux vector in the frame fixed with the stator. In order to estimate the rotor flux components in a desired frame voltage model, current model, full and reduced order observers can be implemented assuming the speed of the motor as an input variable.

The voltage model observer (Jansen and Lorenz, 1994) is based on the integration of the equation of the mathematical model of the motor expressing the dynamics of the stator flux vector and the successive computation of the rotor flux using the equation of the above model which relates the rotor flux to the stator flux and stator current vectors. This observer works well at high speed, whereas at low speed it suffers from the uncertainties in both the knowledge of the stator resistance and the stator voltage measurement.

The current model observer (Takahashi and Noguchi, 1986; Jansen and Lorenz, 1994) is based on the integration of the equation of the mathematical model of the motor expressing the dynamics of the rotor flux vector. It works well at low speed but it is sensitive to the rotor time constant and the magnetizing inductance.

Both the two above observers are combined in a scheme named Improved Gopinath Model (Verghese and Sanders, 1988; Jansen and Lorenz, 1994; Kim, Choi and Sul, 2002).

The full order observers, fourth-order dynamic systems, allows to estimate stator current and rotor flux components from measurements of stator voltage and stator current components (Schreier, et al., 2001; Hinkkanen and Jones, 2002). The principal 
advantage of this observer is the use of the estimated stator currents for processing the control law because they are less noisy than the measured stator currents and do not require filtering which produces time delays in the variables used in the above processing.

The reduced order observers, second-order dynamic systems, allows to estimate only the rotor flux components starting from measurements of the stator current components and speed. Filters of suitable bandwidth can be employed in order to reproduce output signals with negligible delay.

Various structures of reduced order observers of the rotor flux have been obtained using different approaches and assuming that the parameters of the motor assume their nominal values (Alonge and Raimondi, 1987; Bellini, et al., 1988; Verghese and Sanders, 1988; Alonge and Raimondi, 1990). In these papers the choice of the adjustable parameters of the observer is effected, using various methods, in order to obtain a satisfactory dynamic and steady state behaviour also in presence of motor parameter variations.

In the above papers, the tests carried out by simulation have shown that the reduced order observers can be less sensitive to rotor and stator resistance variations and can have a better convergence rate than those based on the implementation of the mathematical model of the rotor circuit, due to their inherent closed loop structure.

In the present work reduced order observers are considered. The design of the observer is carried out in order to satisfy the following design requirements regarding the rotor flux observation error: a) convergence rate; b) low sensitivity to rotor and stator resistance variations; c) low sensitivity to errors due to the implementation of the observers on microprocessor-based systems.

The requirement c) has been introduced in order to take into account the finite processing time of the microprocessor-based systems, which implies a finite sampling period for the observer implementation which causes that the voltages supplying the motor can be very different from those supplying the observer in certain intervals of time, depending on the implementation method employed. It follows that additional oscillations occur in the rotor flux estimation error. In order to reduce these additional oscillations, the difference between the voltages supplying the motor and those supplying the observer is modelled as a disturbance which appears in the mathematical model of the observer itself. In order to take explicitly into account the requirement b) the rotor and stator resistance variations are modelled as a disturbance which appears in the mathematical model of the electromagnetic circuit of the motor. All the requirements a)-c) are then satisfied by minimization of a suitable cost function to minimize the effects of the two above disturbances on the rotor flux observation error, in presence of a constraint which takes into account explicitly the requirement a).

\section{STRUCTURE OF THE OBSERVER}

Neglecting, as usual, the iron losses, the saturation of the electromagnetic circuits and the anisotropy of the geometric structure, and considering explicitly the rotor and stator resistance variations, the mathematical model of the electromagnetic circuit of the induction motor, referred to ab axes fixed with the stator, consists of the following equations (Alonge, D'Ippolito and Raimondi, 2001; cf. also Sangwongwanich, et al., 1990):

$$
\dot{x}=A(\omega) x+B m+G d,
$$

where:

$$
\begin{aligned}
& \boldsymbol{x}=\left[\begin{array}{ll}
\boldsymbol{x}_{1} & \boldsymbol{x}_{2}
\end{array}\right]^{\mathrm{T}}, \quad \boldsymbol{x}_{1}=\left[\begin{array}{ll}
i_{a} & i_{b}
\end{array}\right]^{\mathrm{T}}, \quad \boldsymbol{x}_{2}=\left[\begin{array}{ll}
\hat{\phi}_{a} & \hat{\phi}_{b}
\end{array}\right]^{\mathrm{T}}, \\
& \boldsymbol{m}=\left[\begin{array}{ll}
m_{a} & m_{b}
\end{array}\right]^{\mathrm{T}}, \quad \boldsymbol{d}=\left[\begin{array}{llll}
d_{1} & d_{2} & d_{3} & d_{4}
\end{array}\right]^{\mathrm{T}}, \\
& d_{1}=c_{1} \delta_{s} i_{a}, \quad d_{1}=c_{1} \delta_{s} i_{b}, \quad d_{3}=\frac{\hat{\phi}_{a}}{\delta_{\tau r} L_{e}}, \quad d_{4}=\frac{\hat{\phi}_{b}}{\delta_{\tau r} L_{e}}, \\
& \boldsymbol{A}(\omega)=\left[\begin{array}{ll}
\boldsymbol{A}_{11} & \boldsymbol{A}_{12} \\
\boldsymbol{A}_{21} & \boldsymbol{A}_{22}
\end{array}\right], \quad \boldsymbol{B}=\left[\begin{array}{c}
c_{1} \boldsymbol{I} \\
0
\end{array}\right], \quad \boldsymbol{G}=\left[\begin{array}{c}
\boldsymbol{G}_{a} \\
\boldsymbol{G}_{b}
\end{array}\right], \\
& \boldsymbol{G}_{a}=\left[\begin{array}{ll}
-\boldsymbol{I} & -\boldsymbol{I}
\end{array}\right], \quad \boldsymbol{G}_{b}=\left[\begin{array}{ll}
\boldsymbol{0} & l \boldsymbol{I}
\end{array}\right], \\
& \boldsymbol{A}_{11}=-a_{11} \boldsymbol{I}, \quad \boldsymbol{A}_{12}=a_{13} \boldsymbol{I}-c_{1} \omega \boldsymbol{J}, \quad \boldsymbol{A}_{21}=a_{31} \boldsymbol{I}, \\
& \boldsymbol{A}_{22}=-a_{33} \boldsymbol{I}+\boldsymbol{\omega} \boldsymbol{J}, \mathrm{a}_{11}=\frac{1}{L_{e}}\left(R_{s}+\frac{L_{s}-L_{e}}{\tau_{r}}\right) \\
& l=\frac{1}{c_{1}}, \quad \mathrm{a}_{13}=\frac{1}{\tau_{r} L_{e}}, \quad a_{31}=\frac{L_{s}-L_{e}}{\tau_{r}}, \quad \mathrm{a}_{33}=\frac{1}{\tau_{r}}, \\
& \mathrm{c}_{1}=\frac{1}{L_{e}}, \quad \boldsymbol{I}=\left[\begin{array}{ll}
1 & 0 \\
0 & 1
\end{array}\right], \quad \boldsymbol{J}=\left[\begin{array}{cc}
0 & -1 \\
1 & 0
\end{array}\right],
\end{aligned}
$$

where $\hat{\phi}_{a}$ and $\hat{\phi}_{b}$ are the components of the rotor flux vector $\hat{\phi}$, scaled according to the equation $\hat{\boldsymbol{\phi}}=L_{m} / L_{r} \boldsymbol{\phi}$ being $\boldsymbol{\phi}$ the rotor flux vector, $\delta_{s}$ and $\delta_{r}$ are the stator and the rotor resistance variations and $\delta_{\tau r}=L_{r} / \delta_{r}$. It is convenient to note that $\boldsymbol{d}$ represents a disturbance due to the rotor and stator resistance variations only. 
The problem, now, is to estimate the scaled rotor flux vector $\boldsymbol{x}_{2}$. In this paper, this is effected by means of a reduced order observer. To this end, note that the speed $\omega$ varies slowly with respect to the electromagnetic variables, especially for transportation systems in which the load is practically of inertial type, and consequently it can be considered as an additional input. For this reason the mechanical equation will not be considered here to design the observer. In this case the mathematical model (1) of the electromagnetic circuit of the motor can be considered as a bilinear model.

To solve the above problem the following procedure is employed: a) a structure of Luenberger-type reduced order observer is chosen and expressions of its gain matrices are obtained, as a function of some parameters, in order to force the observation error to converge outside a region which contains the origin $\boldsymbol{e}=\mathbf{0}$, using the Lyapunov approach; c) the parameters are chosen so as to minimize the extension of the above region.

It can be proved the following Assertion.

Assertion - A reduced order observer for the system (1) is given by:

$$
\begin{gathered}
\dot{\boldsymbol{p}}=\boldsymbol{L}(\omega) \boldsymbol{p}+\boldsymbol{K}(\omega) \boldsymbol{x}_{1}+\boldsymbol{B}(\omega) \tilde{\boldsymbol{m}}, \\
\boldsymbol{q}=\boldsymbol{p}+\boldsymbol{K}_{0} \boldsymbol{x}_{1},
\end{gathered}
$$

where:

$$
\begin{aligned}
\boldsymbol{L}(\omega)= & \boldsymbol{L}_{0}+\boldsymbol{L}_{1} \omega, \\
\boldsymbol{K}(\omega)= & {\left[\boldsymbol{L}(\omega)-\boldsymbol{A}_{11}\right] \boldsymbol{K}_{0}+\boldsymbol{A}_{21}-\dot{\boldsymbol{K}}_{0}, } \\
\boldsymbol{B}(\omega)= & -c_{1} \boldsymbol{K}_{0}, \\
& \boldsymbol{L}_{0}=-a_{33} \boldsymbol{I}-a_{13} \boldsymbol{K}_{0}, \\
& \boldsymbol{L}_{1}=\left(\boldsymbol{I}+c_{1} \boldsymbol{K}_{0}\right) \boldsymbol{J},
\end{aligned}
$$

$\boldsymbol{q}$ is the estimate of the rotor flux vector $\hat{\boldsymbol{\phi}}$ and $\tilde{\boldsymbol{m}}$, given by:

$$
\tilde{\boldsymbol{m}}=\boldsymbol{m}+\boldsymbol{\delta} \boldsymbol{m}, \quad \delta \boldsymbol{m}=\left[\begin{array}{ll}
\delta m_{a} & \delta m_{b}
\end{array}\right]^{\mathrm{T}},
$$

takes into account that the voltage supplying the motor is different from that supplying the observer.

To prove the above Assertion it is sufficient to construct the dynamics of the observation error $\boldsymbol{e}(t)=\boldsymbol{x}_{2}(t)-\boldsymbol{q}(t)$ given by:

where:

$$
\dot{e}=L(\omega) \boldsymbol{e}+\boldsymbol{G}_{2} d+\boldsymbol{T}_{2} v
$$

$$
\boldsymbol{G}_{2}=\boldsymbol{G}_{b}-\boldsymbol{K}_{0} \boldsymbol{G}_{a}, \quad \boldsymbol{T}_{2}=\boldsymbol{K}_{0}, \quad \boldsymbol{v}=c_{1} \delta \boldsymbol{m}
$$

Model (6) can be represented as in Fig. 1 which shows that the observer has a feedback structure for $\boldsymbol{K}_{0} \neq \mathbf{0}$ subject to disturbances $\boldsymbol{d}$ and $\boldsymbol{v}$. The problem is the determination of $\boldsymbol{K}_{0}$ in order to minimize the effects of the above disturbances. The method considered in this paper for determining $\boldsymbol{K}_{0}$ is based on the Lyapunov's theory.

Let consider the following definite positive function:

$$
V(\boldsymbol{e})=\frac{1}{2} \boldsymbol{e}^{\mathrm{T}} \boldsymbol{e},
$$

whose time derivative is:

$$
\dot{V}(\boldsymbol{e})=V_{q}+\boldsymbol{d}^{\mathrm{T}} \boldsymbol{G}_{2}^{\mathrm{T}} \boldsymbol{e}+\boldsymbol{v}^{\mathrm{T}} \boldsymbol{T}_{2}^{\mathrm{T}} \boldsymbol{e},
$$

where:

$$
V_{\mathrm{q}}=\frac{1}{2} \boldsymbol{e}^{\mathrm{T}}\left[\boldsymbol{L}(\omega)+\boldsymbol{L}^{\mathrm{T}}(\omega)\right] \boldsymbol{e}
$$

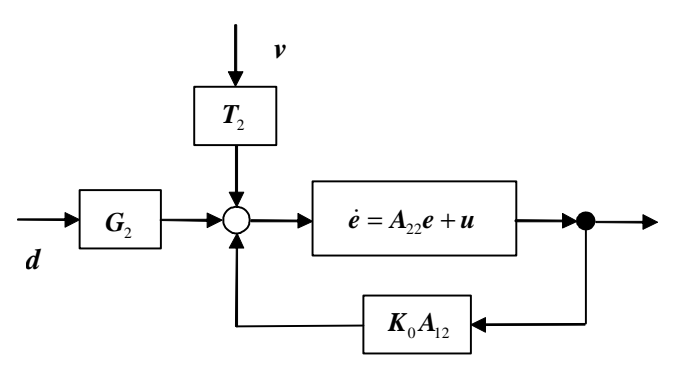

Fig. 1 Block diagram of the dynamics of the rotor flux estimation error.

Substituting in (10) the expression of $\boldsymbol{L}(\omega)$ given in the first of (4), $V_{q}$ becomes:

$$
V_{\mathrm{q}}=\boldsymbol{e}^{\mathrm{T}}\left(\boldsymbol{F}_{0}+\boldsymbol{F}_{1} \omega\right) \boldsymbol{e},
$$

where:

$$
\begin{aligned}
& \boldsymbol{F}_{0}=\frac{1}{2}\left(\boldsymbol{L}_{0}+\boldsymbol{L}_{0}^{\mathrm{T}}\right) \\
& \boldsymbol{F}_{1}=\frac{1}{2}\left(\boldsymbol{L}_{1}+\boldsymbol{L}_{1}^{\mathrm{T}}\right)
\end{aligned}
$$

Assuming, now, $\boldsymbol{d}=\boldsymbol{0}$ e $\boldsymbol{v}=\boldsymbol{0}, \quad \dot{V}(\boldsymbol{e})=V_{q} \quad$ and, consequently, $V_{q}$ must result negative definite; this implies that $\boldsymbol{F}_{0}$ be negative definite and $\boldsymbol{F}_{1}$ be negative definite for $\omega>0$ and positive definite for $\omega<0$. Putting:

$$
\boldsymbol{F}_{0}=-a \boldsymbol{I}, \quad \mathrm{a}>0
$$

and tacking into account the first of (5), the following expression for $\boldsymbol{K}_{0}$ is obtained:

$$
\boldsymbol{K}_{0}=k_{i} \boldsymbol{I}+k_{\boldsymbol{j}} \boldsymbol{J}
$$

where $k_{j}$ will be computed later and:

$$
k_{i}=\frac{a-a_{33}}{a_{13}} .
$$


From (15), (5) and (12) the following expression is obtained for $\boldsymbol{F}_{1}$ :

$$
\boldsymbol{F}_{1}=-c_{1} k_{j} \boldsymbol{I},
$$

which shows that the above conditions on $\boldsymbol{F}_{1}$ are satisfied choosing $k_{j}$ as follows:

$$
k_{j}=r \operatorname{sgn}(\omega), \quad r \geq 0 .
$$

Substituting (17) in (16) and tacking into account (13), (11) becomes:

$$
V_{q}=-f\|\boldsymbol{e}\|^{2}
$$

where:

$$
f=a+c_{1}|\omega|
$$

It follows that:

$$
\dot{V}(\boldsymbol{e})=-f\|\boldsymbol{e}\|^{2}+\boldsymbol{d}^{\mathrm{T}} \boldsymbol{G}_{2}^{\mathrm{T}} \boldsymbol{e}+\boldsymbol{v}^{\mathrm{T}} \boldsymbol{T}_{2}^{\mathrm{T}} \boldsymbol{e},
$$

from which, tacking into account the properties of the norm operator, the following expression is obtained:

$$
\dot{V}(\boldsymbol{e}) \leq-f\|\boldsymbol{e}\|\left[\|\boldsymbol{e}\|-\frac{1}{f}\left(\|\boldsymbol{d}\| \frac{\left\|\boldsymbol{G}_{2}^{\mathrm{T}} \boldsymbol{e}\right\|}{\|\boldsymbol{e}\|}+\|\boldsymbol{v}\| \frac{\left\|\boldsymbol{T}_{2}^{\mathrm{T}} \boldsymbol{e}\right\|}{\|\boldsymbol{e}\|}\right)\right],
$$

which can be written as follows:

$$
\dot{V}(\boldsymbol{e}) \leq-f\|\boldsymbol{e}\|\left[\|\boldsymbol{e}\|-\frac{1}{f}\left(\|\boldsymbol{d}\|_{x} \sigma\left(\boldsymbol{G}_{2}^{\mathrm{T}}\right)+\|\boldsymbol{v}\|_{x} \sigma\left(\boldsymbol{T}_{2}^{\mathrm{T}}\right)\right)\right],
$$

where $\|\boldsymbol{d}\|_{x}$ and $\|\boldsymbol{v}\|_{x}$ are the maximum values of $\|\boldsymbol{d}\|$ and $\|\boldsymbol{v}\|$, respectively, and $\sigma\left(\boldsymbol{G}_{2}^{\mathrm{T}}\right)$ and $\sigma\left(\boldsymbol{T}_{2}^{\mathrm{T}}\right)$ are the maximum singular values of matrices $\boldsymbol{G}_{2}^{\mathrm{T}}$ and $\boldsymbol{T}_{2}^{\mathrm{T}}$.

Equation (19) and the constraints on $a$ and $r$ (cfr. (13) e (17)) show that the function $V(\boldsymbol{e})$ is a Lyapunov's function in the region defined as follows:

$$
\|\boldsymbol{e}\|>\frac{1}{f}\left(\|\boldsymbol{d}\|_{x} \sigma\left(\boldsymbol{G}_{2}^{\mathrm{T}}\right)+\|\boldsymbol{v}\|_{x} \sigma\left(\boldsymbol{T}_{2}^{\mathrm{T}}\right)\right) .
$$

Consequently, the choice of the function (8) as candidate Lyapunov's function assures the convergence of the observation error to a minimum value given by (20). In order to reduce this error it is convenient to determine $a$ and $r$ so as to minimize the following cost function:

$$
J_{c}=p_{1} \frac{\sigma^{2}\left(\boldsymbol{G}_{2}^{\mathrm{T}}\right)}{f^{2}}+p_{2} \frac{\sigma^{2}\left(\boldsymbol{T}_{2}^{\mathrm{T}}\right)}{f^{2}}
$$

subject to the constraints:

$$
\begin{aligned}
& a \geq a_{0}>0, \\
& r \geq r_{0} \geq 0 .
\end{aligned}
$$

Obviously, assuming $p_{1}=1$ and $p_{2}=0$ the effects of $\boldsymbol{d}$ are minimized, whereas putting $p_{1}=0$ and $p_{2}=1$ the effects of $\boldsymbol{v}$ are minimized. The solutions of the above constrained minimization problem are obtained using the sufficient Kuhn-Tucker conditions.

Assuming $a>a_{0}$ and $r>r_{0}>0$ the following solution is obtained:

$$
\begin{aligned}
& a=a_{33} \\
& r=\frac{\rho}{a_{13}}|\omega|
\end{aligned}
$$

where:

$$
\rho=\frac{p_{1}}{p_{2}+2 p_{1}}
$$

The behaviour of this observer, tested by simulation, is not satisfactory; this is due to the very high values of the elements of matrices $\boldsymbol{G}_{2}$ and $\boldsymbol{T}_{2}$.

Assuming $a=a_{0}$ e $\mathrm{r}>\mathrm{r}_{0}>0$, the solution is:

$$
\begin{aligned}
& a=a_{0} \\
& r=\frac{c_{1}}{a_{0}}\left(k_{i 0}^{2}+2 \rho k_{i 0} l+\rho l^{2}\right)|\omega|
\end{aligned}
$$

where:

$$
k_{i 0}=\frac{a_{0}-a_{33}}{a_{13}} .
$$

Also in this case the behaviour of the observer is not satisfactory for the same reasons as before.

Assuming $a>a_{0}$ and $r=0$ it is obtained $\boldsymbol{F}_{1}=\boldsymbol{0}$ and $\boldsymbol{K}_{0}=\mathbf{0}$ which implies that the observer coincides with the equations of the rotor circuit, i.e. the current model observer. As already said, the behaviour of this observer is not satisfactory.

Finally, assuming $a>a_{0}$ and $r=r_{0}>0$ the following solution is obtained:

$$
\begin{aligned}
& a=a_{33}(1-\rho) \frac{a_{33}+c_{1} r_{0}|\omega|}{a_{33}(1-\rho)+c_{1} r_{0}|\omega|}, \\
& r=r_{0}
\end{aligned}
$$

which implies that $\left|k_{i}\right|$ increases with $\omega$ and assumes the value $\rho a_{33}$ at high speed, whereas $k_{j}$ is independent of $\omega$. It follows that the elements of 
$\boldsymbol{G}_{2}$ e $\boldsymbol{T}_{2}$ do not increase with $\boldsymbol{\omega}$. This solution has been chosen and utilized in the paper.

\section{EXPERIMENTAL RESULTS}

In order to test the behaviour of the observer, experiments have been carried out on a prototype consisting of a $2.2 \mathrm{~kW}$ induction motor supplied by a voltage source inverter, a measurement system employing two Hall current transducers and a 1024 ppr incremental encoder for measurements of two stator currents and speed, respectively, and a dSpace DS1103 microcontroller for the implementation of a simple PI-based controller and the proposed observer and for data acquisition. The implementation of the observer on the above digital system has been carried out using the second-order Runge-Kutta numerical method of integration of differential equations, which is now supported by the last generation DSP boards. This method gives more accurate results than the Euler method, but it requires higher computation times and, consequently, higher sampling periods.

The nominal values of the parameters of the motor useful for the observer design, identified using the approach illustrated in (Alonge, D'Ippolito and Raimondi, 2001), are given by:

$$
\begin{gathered}
T_{r}=0.1586 \mathrm{~s}, R_{s}=2.9673 \Omega, \\
L_{s}=0.37686 \mathrm{H}, L_{e}=0.02555 \mathrm{H},
\end{gathered}
$$

Other data relative to the experimental setup are:

\begin{tabular}{|l|l|}
\hline DC link voltage & $540 \mathrm{~V}$ \\
\hline Maximum current & $10 \mathrm{~A}$ \\
\hline Hall current transducers & $\pm 5 \mathrm{~V} / \pm 50 \mathrm{~A}$ \\
\hline Sampling freq. (data acquisition) & $12 \mathrm{kHz}$ \\
\hline Sampling freq. (PWM) & $12 \mathrm{kHz}$ \\
\hline$p_{1}$ & 0.8 \\
\hline$p_{2}$ & 0.2 \\
\hline$r$ & 0.002 \\
\hline
\end{tabular}

In order to test the observer, the stationary frame mathematical model has been implemented on the above DS1103 digital system and the rotor flux computed using it is compared with the rotor flux estimated by means of the observer.

The experimental results are shown in Figs. 2 and 3; these Figures have been obtained using MATLAB starting from data acquired by the DS1103 system. Fig. 2 refer to a transient corresponding to a step variation of the reference speed from 0 to $30 \mathrm{rad} / \mathrm{s}$. Fig. 3 correspond to a step variation of the reference speed from 0 to $140 \mathrm{rad} / \mathrm{s}$ followed from a speed inversion from 140 to $-140 \mathrm{rad} / \mathrm{s}$.
Examination of these Figures shows that the observed rotor flux is practically the same of the rotor flux computed from the model processed in real time by the above DSP-based system together with the controller and the observer. In particular, for the experiments considered in Fig. 2 and 3 , the maximum absolute errors are 0.0015 and $0.008 \mathrm{~Wb}$, respectively.

\section{CONCLUSIONS}

The observer considered in this paper allows to minimize the effects of the rotor and stator resistance variations and the differences of the supply voltage of the motor and that of the observer.

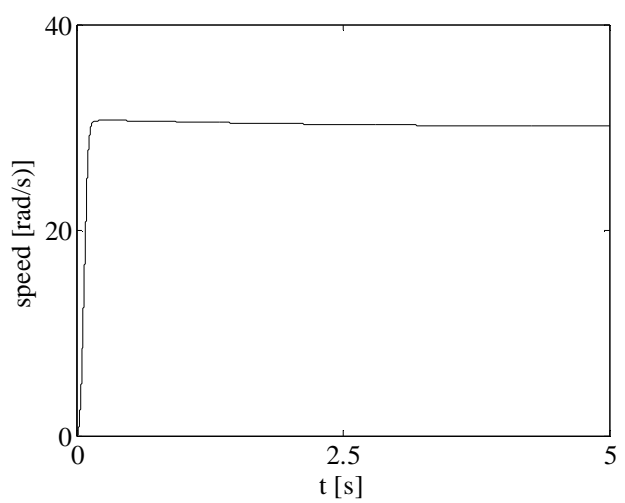

a)

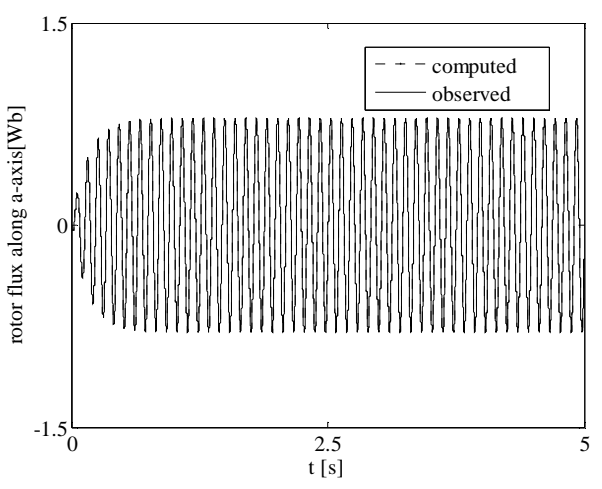

b)

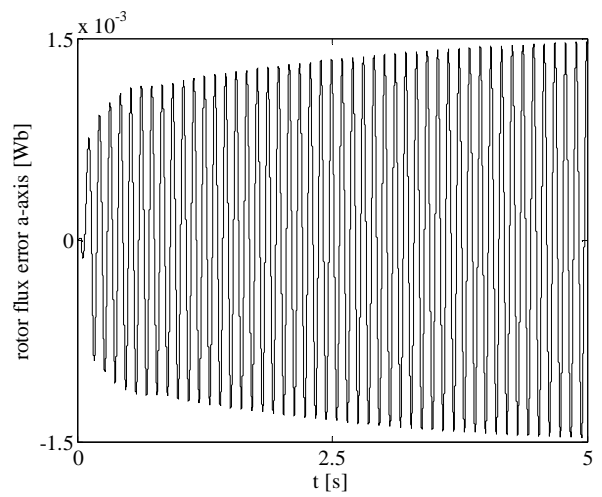

c)

Fig. 2 Transient and steady-state behaviour of the reduced-order observer: reference step variation from 0 to $30 \mathrm{rad} / \mathrm{s}$. 
Several tests carried out by simulation in various operating situations have shown a low sensitivity regard all the parameters of the motor. The experimental results carried out on a prototype show that the observer displays a good behaviour also at low speed. The observer can be easily implemented on a floating point DSP. For practical implementation on a fixed point DSP, the problem due to the complexity of the observer can be overcome using look-up tables. This problem will be object of future work.

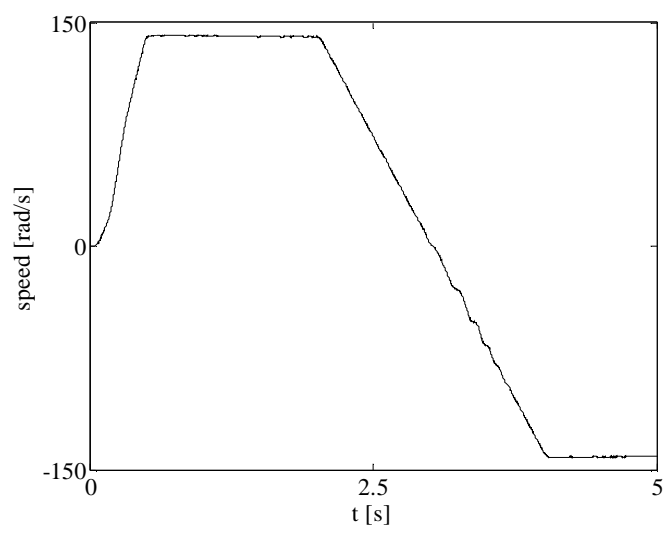

a)

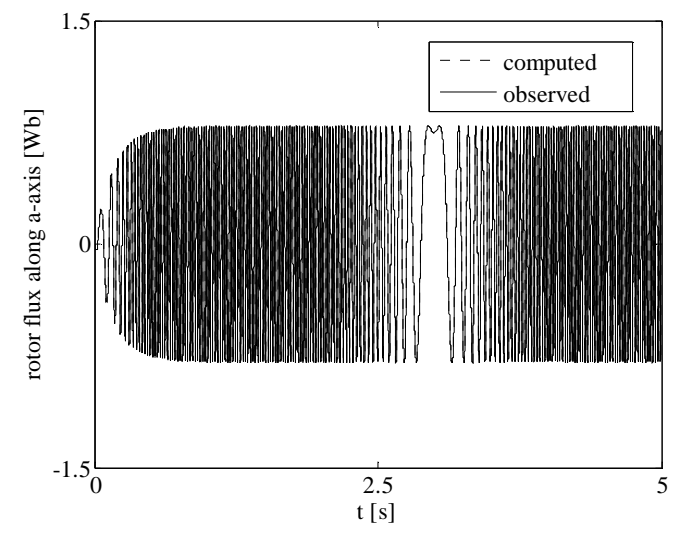

b)

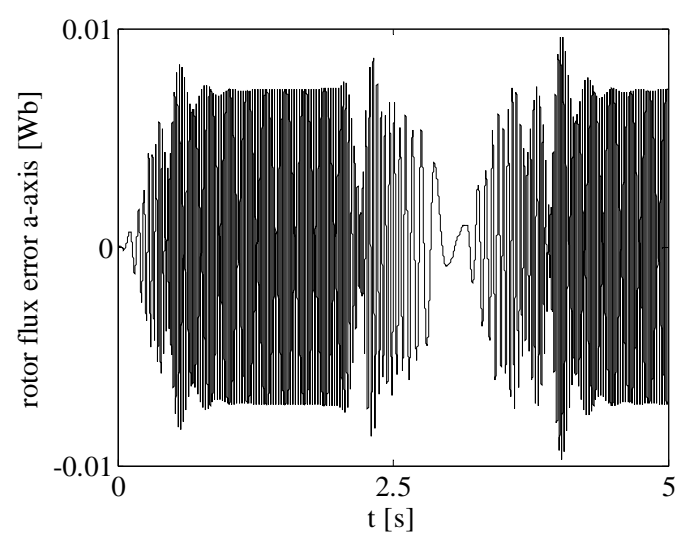

c)

Fig. 3 Transient and steady-state behaviour of the reduced-order observer: reference step variation from 0 to $140 \mathrm{rad} / \mathrm{s}$ followed from a speed inversion from 140 to -140 .

\section{ACKNOWLEDGEMENTS}

This work was partially supported by the University of Palermo (ex 60\%).

\section{REFERENCES}

Jansen, P. L., Lorenz, R. D. (1994). A Phisically Insightful Approach to the Design and Accuracy Asseeement of Flux Observers for Field Oriented Induction Machine Drives. IEEE Trans. on Industry Applications, Vol. 30, 101 110.

Takahashi, I. and T. Noguchi (1986). A new quickresponse and high-efficiency control strategy of an induction motor. IEEE Trans. on Industry Applications, Vol. 30, 101 - 110.

Schreier, G., J. DeLeon, A. Glumineau and R. Boisliveau (2001). Cascade Nonlinear Observers: Application to an Experimental Induction Motor Benchmark. IEE Proc. Control Theory and Appl., Vol. 148, 509-515.

Kim, J. H., Choi, J. W. and S. K. Sul (2002). Novel rotor-flux observer using observer characteristic function in complex vector space for fieldoriented induction motor drives. IEEE Trans. on Industry Applications, Vol. 38, 1334 - 1343.

Hinkkanen, M. and J. Luomi (2002). Parameter Sensitivity of Full-order Observers for Induction Motors. IEEE Trans. on Industry Applications, Vol. 39, 1127 - 1135.

Alonge, F. and T. Raimondi (1987). Design of a reduced order observer for induction motors. In Proc. 7th Int. Conf. on Control Systems and Computer Science, Vol. 1, 46-55.

Bellini, A., G. Figalli and G. Ulivi (1988). Analysis and design of a microcomputer-based observer for an induction motor. Automatica, Vol. 24, 549-555.

Verghese, G. C. and S.R. Sanders (1988). Observers for flux estimation in induction machines. IEEE Trans. on Ind. Electron., Vol. 35, 85-94.

Alonge, F. and T. Raimondi (1990). An estimator of rotor flux of induction motors. In Proc. 6th Conf. on Power Electronics and Motion Control, Vol. 2, 494-499.

Sangwongwanich, S., T. Yonemoto, T. Furuhashi and S. Okuma (1990). Design of sliding observers for robust estimation of rotor flux of induction motors. Proc. IPEC-Tokyo Conference, Vol. 2, 1235-1242.

Alonge, F., F. D'Ippolito and F. M. Raimondi (2001). Least Squares and Genetic Algorithms for Parameters Identification of Induction Motors. Control Engineering Practice, Vol. 5, 32-41. 